Ultra-Wideband, Short-Pulse Electromagnetics 5 edited by P.D. Smith and S.R. Cloude, November 2002, 199(8)

\title{
UWB SUBSURFACE RADAR WITH ANTENNA ARRAY FOR IMAGING OF INTERNAL STRUCTURE OF CONCRETE STRUCTURAL ELEMENTS
}

\author{
Anatoliy O. Boryssenko, Elena S. Boryssenko, Vladimir A. Ivashchuk, \\ Alexander N. Lishchenko, Vitaliy P. Prokhorenko \\ Research Company "Diascarb" \\ Kyiv, P.O. Box No. 222, 02222, Ukraine
}

\section{INTRODUCTION}

The basic design principals implemented in the advanced UWB radar with timedomain operation and with the $100-900 \mathrm{MHz}$ effective band are considered including real/synthetic array and full-polarimetric schema. The radar is installing on a moveable platform for remote operation on the territories with radioactive pollution. This probing system should be employed near the nuclear power plant block No. 4 of the Chernobyl nuclear power plant near Kyiv, Ukraine, damaged in 1986. The key task is searching and localisation of the nuclear-fuel contained materials (NFCM) inside the Cascade Wall around nuclear reactor. The testing of the presented UWB radar is in progress now.

\section{PROBLEM BACKGROUND}

Basically the problem to locate NFCM inside the concrete Cascade Wall can be solved by nonstandard ground penetrating radar (GPR) technique rather than by commercially available radars. The challenger features of the treated problem involve:

i) sounding medium is thick up 10 meters and more layered concrete medium;

ii) this medium has strong signal attenuation depended on internal water/ionic content;

iii) it is inhomogeneous medium due to chaotic internal filling with a large variety of different objects including elements of the destroyed reactor dispersed by explosion;

iv) NFCM should be detected can be as solidified masses of molted concrete with patches of radioactive substances;

v) it is expected that in some cases those patches may have a cylindrical shape as original fuel-assemblies;

vi) searching areas on the horizontal plane of the Cascade Wall have rectangular shape where edge regions near vertical walls will cause strong diffraction interference (figure 1); vii) only completely automatic radar operation can be possible due to radioactive pollution of searching areas. 


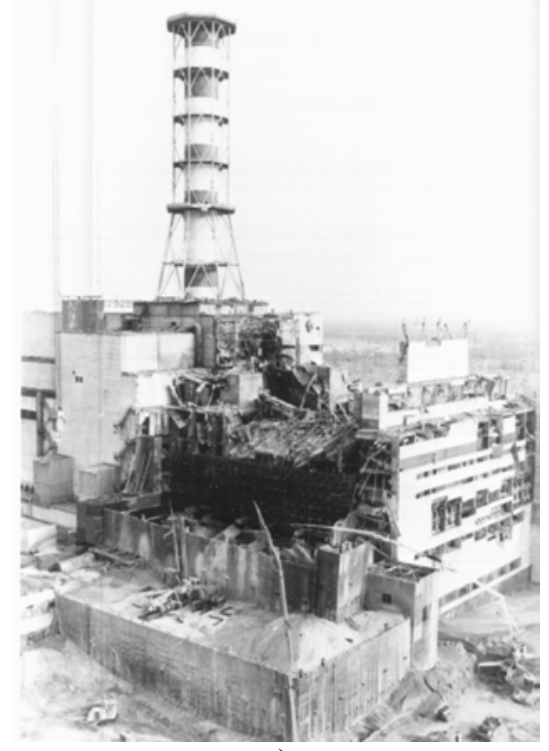

a)

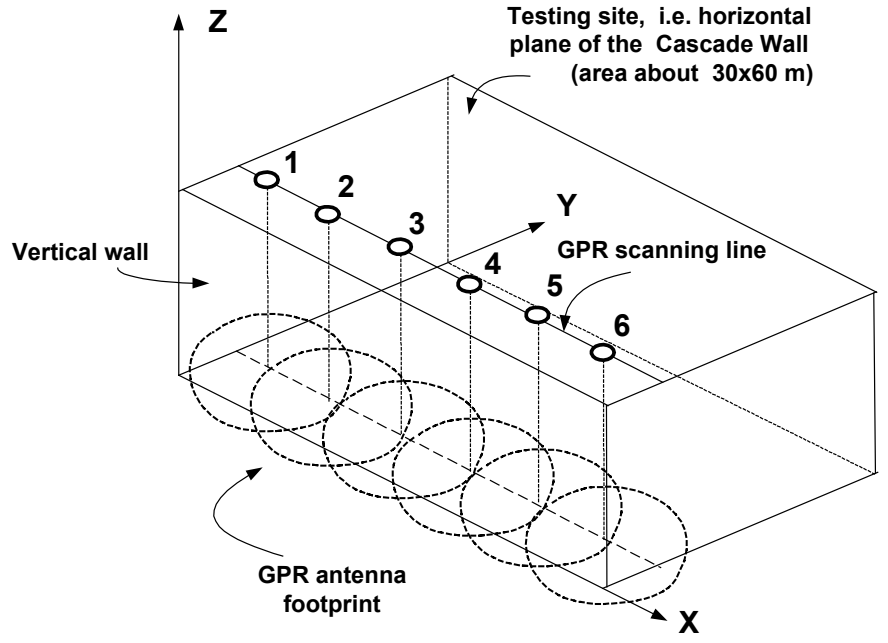

b)

Figure 1. The Cascade Wall the Chernobyl NNP Unit No. 4.: (a) Photo of test site during the nuclear disaster http://www.chernobyl.com. (b) Schematic sketch of GPR survey and its limitations on the horizontal planes.

Actually any GPR maps the changes within material under survey due to contrasts in the electromagnetic properties. So NFCM can be recognized inside concrete by a set of its inferential features. For the most of known GPR applications the measured data are presented as distorted images or pseudo-cross-sections of the subsurface regions for visual analysis, without any further necessity of processing (Olhoeft, 2000). However, the considered problem requires more detailed and precise information about type of each hidden object, its depth, orientation, composition etc. that can be obtained by properly acquiring the data with advanced subsurface radar.

One can find some useful approaches to be applied here in the such fields of GPR applications like land-mine and UXO detection (Daniels, 1999), nondestructive testing of concrete in civil engineering (Boryssenko et al, 2000) and for noninvasive profiling of asphalt and concrete pavement and bridge desks (Wahrus et al, 1994).

Key problems to solve, when GPR is applied for land mine detection, are connected with small electrical contrast of mines like plastic ones and influence of environment on radar performances. The last is due to irregular stratified structure of sounding media, rough interfaces, the presence of clutters like tree roots, rocks etc. Inspection of concrete structures with microwave is powerful tool with a wide range of applications. This is a rapid technique for nondestructive detection of defects in reinforcement concrete, for multilayered road structure and so on.

Note that all listed above application are connected with concrete probing up to depth of several meters while the considered problem requires more deep down-range operation under specific conditions.

We conclude that for successful detection and discrimination of the FCM objects deeply inside the concrete construction of the Cascade Wall can be reached by application of the advanced GPR rather than ordinary hand-held GPR. Such advanced GPR should possess a high dynamical range and optimal operating frequency band. Also physical aperture technique should be employed beyond the synthetic aperture method. Polarimetric and other signal processing approaches have to be incorporated in this radar project. High procession of antenna space positioning is also required for exact subsurface mapping. The last can be fulfilled by robotic platform where radar is housed and that is urgently needed 
Ultra-Wideband, Short-Pulse Electromagnetics 5 edited by P.D. Smith and S.R. Cloude, November 2002, 199(8)

for safety operation on the area with radioactive pollution. Some of the formulated above features of the presented GPR project are under consideration in this paper.

\section{GENERAL DESIGN APPROACHES}

The key principal employed in this design is that such system must be realised in the short term with available technologies and examined before solutions. The presented radar possesses features originated from previous research and design projects in UWB radar especially for subsurface probing based on the time-domain technology.

\section{Optimal Frequency Band}

The key factor should be care is electrical properties of concrete as medium for electromagnetic wave propagation. Those properties have been studied by many researchers in $1 \mathrm{MHz}-10 \mathrm{GHz}$ band (Robert, 1998). Figure 2 summaries those data and illustrates the high frequency limit for GPR probing in concrete. Generally there exists a common tradeoff between resolution and depth range for choice of GPR operation frequency band. The increasing of resolution, using of high frequencies, can be achieved by the price to pay as dramatic rise of radar potential and appearance on radar images the redundant details and speckle structure (Daniels, 1999). There is also low frequency limit for optimal detection of target with specific shape and size like expected NFCM to minimize signal-to-clutter ratio besides requirement of high resolution (Brock and Patitz, 1994). Due to above reasons the $100-900 \mathrm{MHz}$ band is optimal one for the designed GPR.

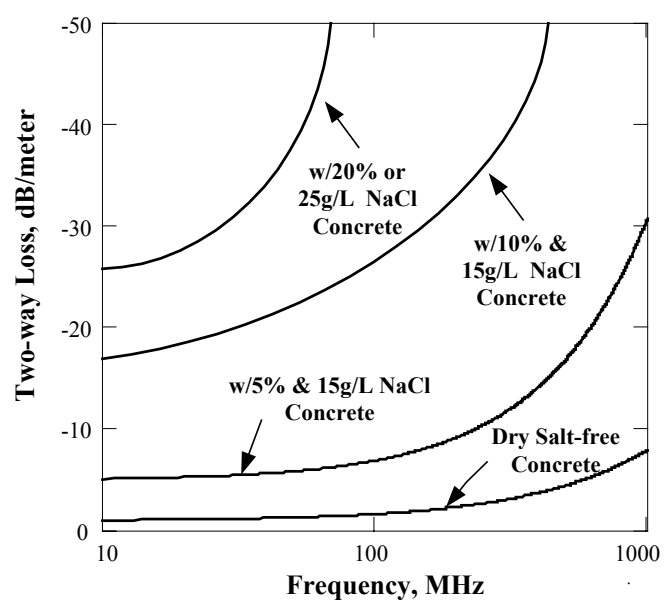

Figure 2. Dispersion properties of concrete attenuation factor versus operation frequency band and internal water / ionic $(\mathrm{NaCl})$ content.

\section{Radar Energy Potential}

Here we express the radar performance factor as the usual ratio of the peak radiated power to the smallest detectable receiver signal (Wahrus, et al,1994; Daniels, 1999). Whereas the first factor is a constant value, additional coherent processing including averaging in time and space can increase the second one.

Impulse generator of transmitter forms $2000 \mathrm{~V}_{\mathrm{p}-\mathrm{p}}(56 \mathrm{~dB})$ bell-like pulse on the 10 Ohm impedance antenna as a load. The minimum discernible signal $(M D S)$ is expressed as (Wahrus, J. P., et al, 1994): $M D S=k \cdot T \cdot B W \cdot N F \cdot S N R$, where $k$ - Boltzmann constant, $T$ maximum operation temperature, $B W=800 \mathrm{MHz}$ - bandwidth, $N F$ - Noise Factor of the receiver, $S N R$ - Signal-to-Noise Ratio. Assume that $N F=1.3 \mathrm{~dB}$ and $S N R=15 \mathrm{~dB}$. At this 
Ultra-Wideband, Short-Pulse Electromagnetics 5 edited by P.D. Smith and S.R. Cloude, November 2002, 199(8)

case we have $M D S=-96 \mathrm{~dB}$. We consider peak power rather energy because in highresolution radar system shape of transmitting signal is precisely registered and processed.

Additional gain of system performance factor is following as stated above from radar response coherent processing like averaging in time with factor up to 2048 (33dB), array processing for physical aperture $(9 \mathrm{~dB})$ and synthetic aperture $(12-20 \mathrm{~dB})$. Therefor the total energetic potential reaches of $180 \mathrm{~dB}$ magnitude. Sufficient rise of the last figure by processing is possible due to radar operation with discrete profiling when system has high level of stationary determined by platform positioning at each measurement point.

The high system energy potential enables high depth range of radar. In this case for typical 5\% internal water content in accordance to Figure 1 the two-way loss magnitude is about $12 \mathrm{~dB}$ per meter. The last corresponds to depth range of about $15 \mathrm{~m}$ that give opportunity to search through all thickens of the Cascade Wall.

\section{Radar signals}

We paid particular attention to optimising transmitter/receiver array subsystems including optimal choice of signal waveforms and equivalent effective spectra shown in figure 3. Transmitting antenna driving pulse has a rise time of $1 \mathrm{~ns}$ and 5 -ns fall time. The radiated electromagnetic signal is determined by the current excitation waveform, the antennas and ground-coupling factor mainly. The last has stochastic disturbance effect on both the transmitter and receiver radar antennas resulted in registered waveform distortion. Also effect of multiple cooperative /non-cooperative scattering (Astanin et al, 1994) by specific shape targets will further modify the received signal making its different form idealised one in figure $3 \mathrm{c}$. Other factors change the registered signal waveform including: roughness of surface interface, antenna shielding from side of upper half-space, nonuniformity of antenna patterns at the angles off vertical directions etc. Finally the common measured two-pass waveform is depicted in figure 4.
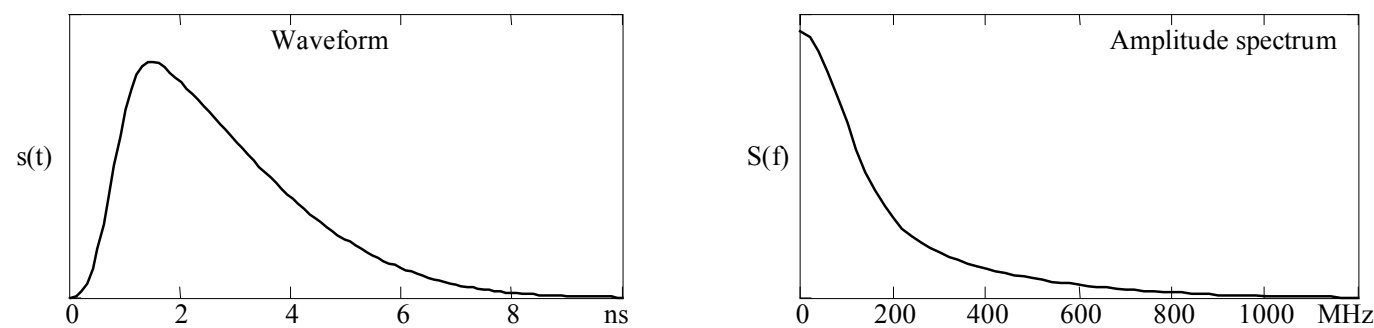

a)
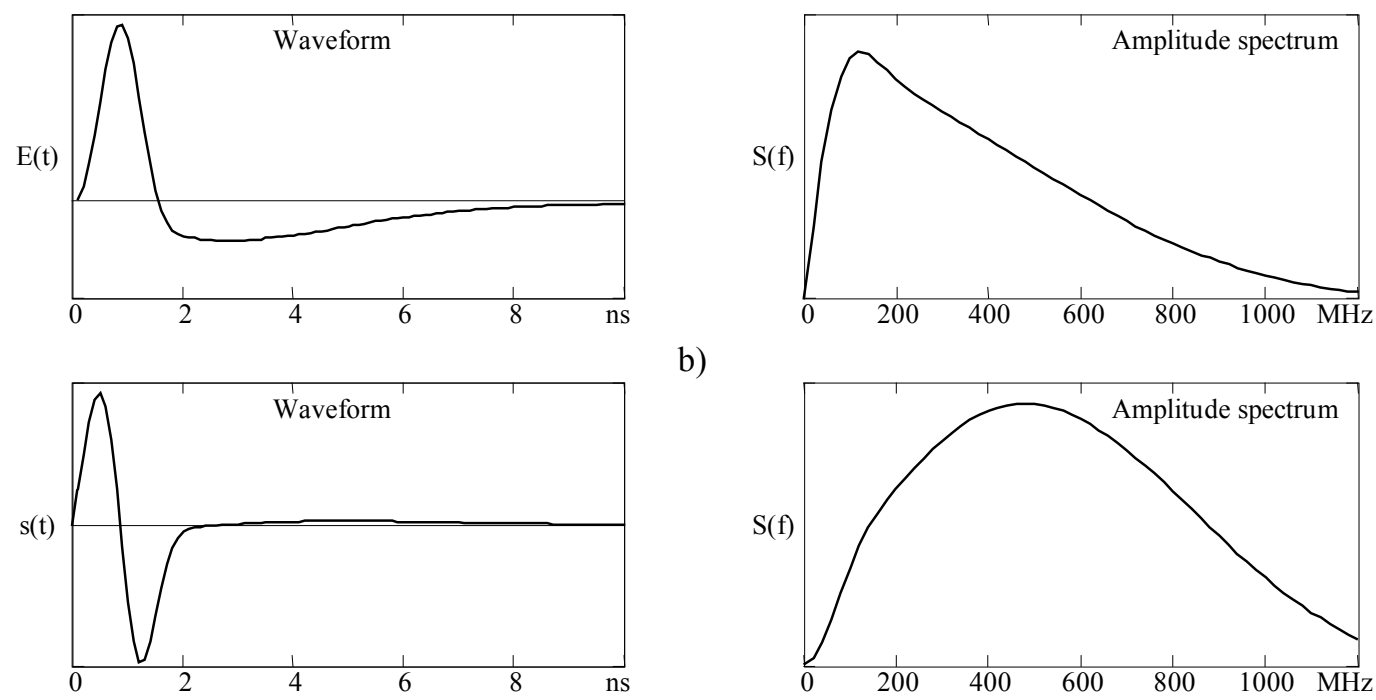

b)

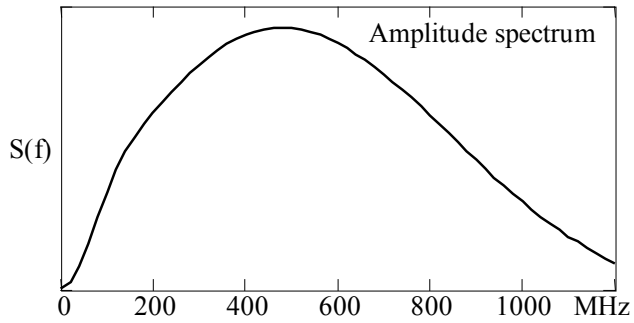


Ultra-Wideband, Short-Pulse Electromagnetics 5 edited by P.D. Smith and S.R. Cloude, November 2002, 199(8)

c)

Figure 3. Waveforms and corresponding amplitude spectrum of (a) transmitter output pulse to driver antenna and (b) radiated wavelet and (c) received waveform.

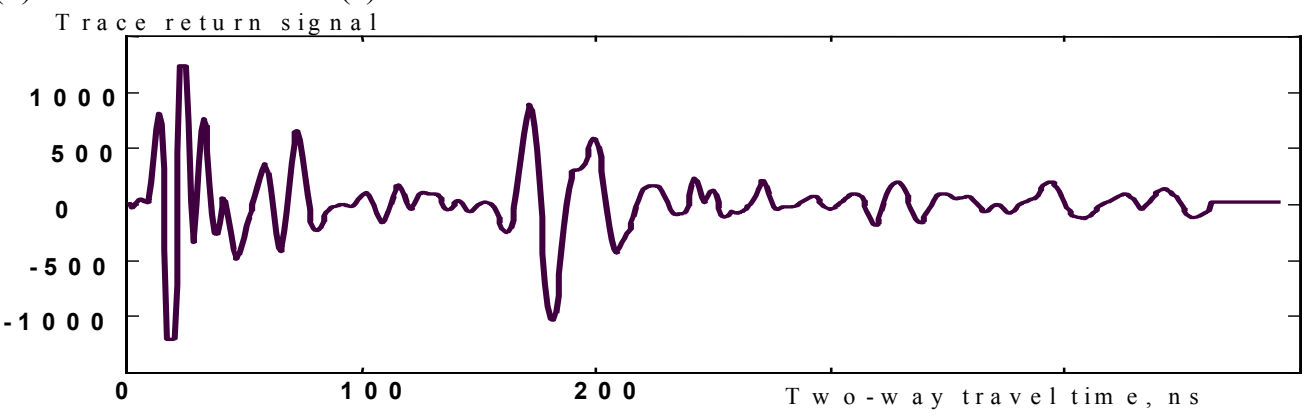

Figure 4. Real two-pass signal registered in transmitter-medium-receiver channel.

\section{SYSTEM ARCHITECTURE OVERVIEW}

Generally the presented UWB radar system includes two principal components, i.e. electronic and mechanical ones, the multi-channel radar and movable robotic platform. The multi-channel UWB radar consists of three main subsystems: 1) sensor receiving array; 2) transmitter/receiver electronics; 3) data and command transfer via optical line, 3) operation control and data processing/storing.

Transmitter electronics is based on the drift step recovery diode diodes and special driving circuits. Lower pulse repetition rate (PRT) are employed in this radar for the reason to increase life time of compact high-voltage transmitter units, to maintain its optimal temperature regime for stable operation without external cooler and for power supply minimization.

Receivers are built with using low-noise input circuits and stroboscopic time-domain sampling as for conventional pulse GPR. This approach and 16 digit ADC circuit enable high-accuracy registration of time-scaled signal waveforms. Such operation require some time with low PRT and long signal averaging but is not drawback for the presented system because total data collection time at each point of scanning line is lower that time required for movement and positioning of mechanical part of radar.

Radar control and data storing by the remote central PC are fulfilled by the data/command transmission subsystem. The last forms a local network between computer on robotic platform and the central computer installed on the safe distance up to $0.5 \mathrm{~km}$ from the working zone of radar. Physical interface of the data exchange between GPR and control PC unit is based on fiber optic communication link with two-way data transfer channels possibility.

The radar sensor array is installed on the robotic platform for remote operation. The robotic platform is in fact a controlled distantly carrier of radar for its programmed replacement over searching territory. In process of radar survey the sensor array is moving in discrete mode over the searching area. We employed as basic for robotic platform design electrical and pneumatic module components of the firm FESTO.

Table 1 lists the principal characteristics of UWB advanced subsurface radar.

Table 1. List of the UWB radar principal features.

\begin{tabular}{|l|l|}
\hline Signal: asymmetric monopulse (figure 3) & Time Averaging Factor: up to 33dB \\
\hline Effective Bandwidth: $100 \ldots 900 \mathrm{MHz}$ & Array Averaging Factor: up $29 \mathrm{~dB}$ \\
\hline Energetic Potential: $180 \mathrm{~dB}$ & Number of Transmitter Channel: 2 \\
\hline
\end{tabular}


Ultra-Wideband, Short-Pulse Electromagnetics 5 edited by P.D. Smith and S.R. Cloude, November 2002, 199(8)

\begin{tabular}{|l|l|}
\hline MRS: $-96 \mathrm{~dB}$ & Number of Receiver Channel: 8 \\
\hline Transmitter Peak Voltage: 2000 Volt & Number of Polarisation State: 2 \\
\hline Pulse Repetition Rate $: 6 \mathrm{kHz}$ & Operation Life: 1200 hours \\
\hline
\end{tabular}

\section{ANTENNA ARRAY AND PROCESSING TECHNIQUES}

Inherently the beam pattern of subsurface radar antennas is widely spread (Daniels, 1999) and to improve it the synthetic aperture technique (SAR) technique (Soumekh, 1999 ) is widely employed. The SAR is based on moving of radar antennas along scanning line laid over searching area (Finkelstein, 1994). In this way processing of radar data forms image of subsurface region.

Actually migration processing (Morah, 2000) is basic imaging technique, which rearranges reflected data so that reflection and diffraction events are plotted at the origin locations. Effectiveness of the SAR depends on radar performances and specific features of sites under radar survey. For the considered problem the most unfavorable factor is limited size of the horizontal plane of searching areas due to the edge effects on SAR as shown conditionally in figure 1 .

In order to overcome limitations of existence GPR technique, such as for hand-held radar, the physical aperture combined with accurate SAR should be employed. In this way a near-field beam-forming with real physical aperture array is implemented by adjusting time delay magnitudes in receiving channels for array focusing on definite space point (spot) inside volume covered by antenna array (Rappaport and Reidy, 1994). Array structure, in figure 5a, implements a high-resolution post-processing array method for radar imaging with improving of signal-to-noise ratio, down-look radar range and suppers interference signal with out of interest arriving.

Main functional goal of radar on the survey site is detection and discrimination of NFCM as stated before. In the case of target with definite geometrical shape the value of scattered response will depend on target position with respect to polarization. Reliable radar system should be robastic to this factor and designed to separate transmitter and receiver polarization states for arbitrary located buried target. In another words, the polarimetric radar measurement schema is urgently required.

Figure $5 \mathrm{~b}$ shows the dual polarized radar array antenna with separated two transmitting and eight receiving antennas. Besides target characterization radar polarimetric technique improves quality of radar image of subsurface region by reducing its speckle components (Stiles et al, 1999). There are two basic configurations, i.e. Vivaldi tapered slot antenna and TEM ridge horn antenna. All antennas are resistively loaded for prevent ringing antenna effect and optimise signal waveform.

The transmitter and receiver modules are directly terminated to elements of antenna array. The radar array has upper shielding for improving system interference immunity. 
Ultra-Wideband, Short-Pulse Electromagnetics 5 edited by P.D. Smith and S.R. Cloude, November 2002, 199(8)

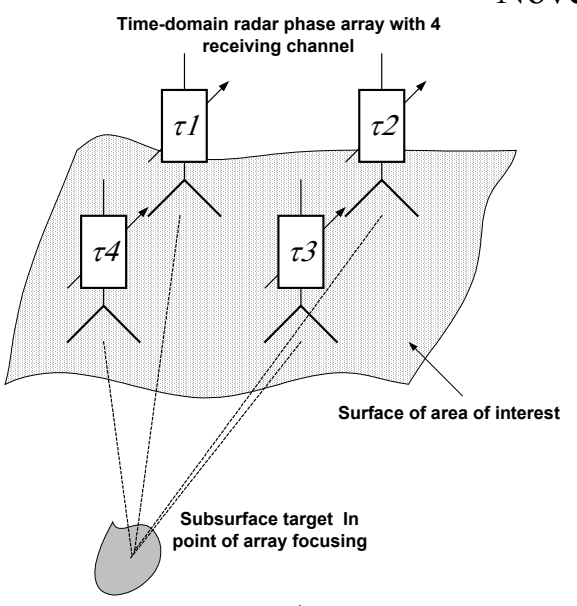

a)

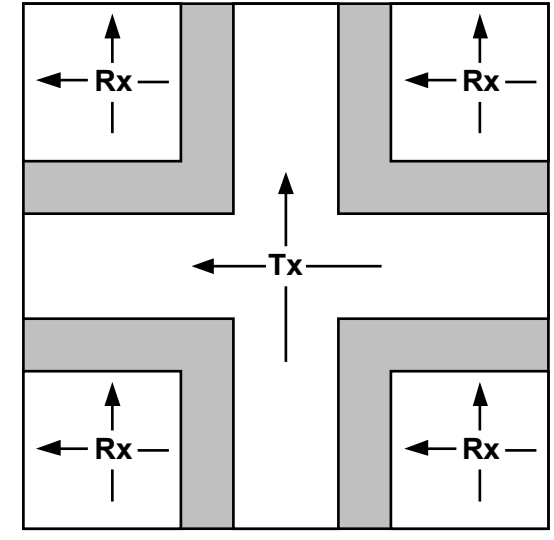

b)

Figure 5. (a) Antenna array time-domain beam-forming by cross-correlation of channel signals with properly introduced time delay $\tau 1 \ldots \tau 4$. (b) Schematic presentation of complete antenna array for two polarization states of transmitters Tx and receivers $\mathrm{Rx}$.

\section{DATA COLLECTION AND PROCESSING}

The presented radar system implements the following in situ radar measurement that based on above described principal moments. Robotic transport platform after installation, mounting radar on it and termination to external power, control and data transfer subsystems begins operation. In process of operation platform with radar is moving in step mode for discrete probing. Total trajectory of movement over searching area will cover it by the set of scanning lines.

At each step the radar array unit is precisely positioned on surface. The plane coordinates of antenna reference point is measured by platform positioning subsystems and fixed in data file of the measurement records. Then radar measurement is accomplished consequently for both polarizations of transmitters when all eight receivers are working to register the scattered signal. Measurement at each point can be accomplished with different scenario of radar operation including time-varying gain control, data averaging, nonuniform sampling and so on. The central remote PC unit stores results of measurement at each point with coordinate and radar-setting information.

Radar team can visualize data received at each point that based high-resolution antenna array technique with time-domain beam forming. In this way searching area covered by antenna physical aperture can be preliminary visualized in the 2-D or 3-D form. Next the transport platform should move along given scanning line and measurement procedure described above would be repeated.

Much of the effectiveness of GPR technology is a function of the skill of the GPR team including operator and data interpreter. GPR equipment must be designed to provide in situ maximum effectiveness of radar data collections. Radar data are analyzed from a graphical presentation in the 2-D forms like of the B-scan (vertical section) and C-scan (horizontal section) or in 3-D from. Those graphical representations contains focused images of subsurface medium showing its various features originated from the contrast of electrical properties, i.e. dielectric constant, conductivity and magnetic constant rarely. Other factors governing features of scattered signals are attenuation, RCS, polarization etc. Each subsurface scattering phenomenon is characterized by a set of those parameters. The last is not complete unique and used actually for the probabilistic discrimination of the following elements of subsurface medium inside the concrete Cascade Wall.

For illustration figure 6 simulated (left) and real field result (left) for focused radar 2D image of subsurface scatterer like reinforcement bar in concrete. 
Ultra-Wideband, Short-Pulse Electromagnetics 5 edited by P.D. Smith and S.R. Cloude, November 2002, 199(8)
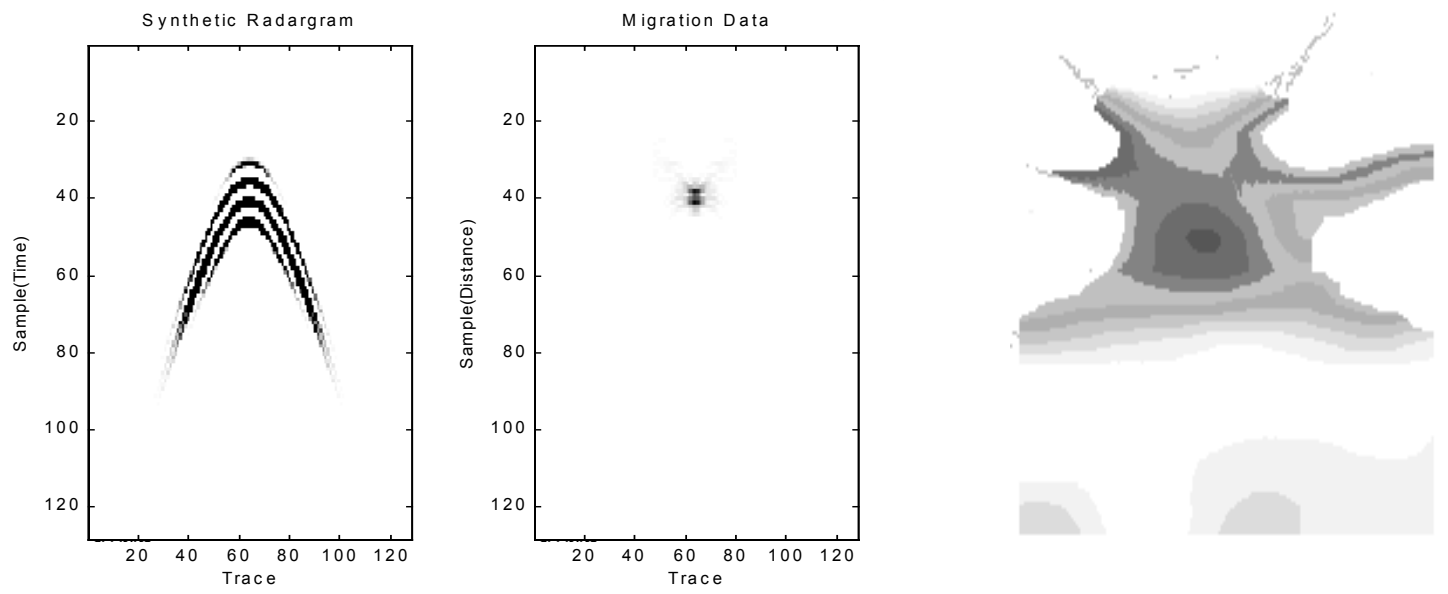

a)

b)

Figure 6. (a) Simulated synthetic aperture image of long small-diameter cylindrical subsurface target. (b) Focused images of reinforcement bar inside concrete registered by GPR with 2-receiver array.

\section{CONCLUSION}

Several recent all the principal components of the presented radar system have been finished in manufacturing and will be tested. First the radar with two-receiver array for copolar and cross-polar operation has been examined. The most resent experiments involve four-receiver array for the signal processing algorithms with TD beam forming. Next will include full radar installation on platform for remote operation. At the moment of this paper preparation the final decision about using of such GPR system was unknown in the frame of the International Shelter Project (SIP). If this decision will be positive the radar system will be assembled completely on the robotic platform for its final testing, updating and filed changing.

\section{REFERENCES}

Astanin, L. Yu., Kostylev A. A., Zinoviev Yu. S., Pasmurov A. Ya., 1994, Radar Target Characteristics: Measurement and Applications, CRC Press, Boca Raton.

Boryssenko, A. A., Boryssenko, E. S., Lishchenko, A. N., Prokhorenko, V. P., 2000, Inspection of Internal Structure of Walls by Subsurface Radar, Submitted to 2000 GPR International Conferernce, Queensland, Australia.

Brock, B. C., Patitz, W.E., 1994, Factors Governing of Operation Frequency for Subsurface-Imaging Synthetic-Aperture Radar, in: Proceedings of SPIE Conference, 2217: 176.

Daniels, D. J., 1999, System Design of Radar for Mine Detection, in: Proceedings of SPIE Conference on Subsurface Sensors and Applications, 3752:390.

Finkelstein, M.I, Ed., 1994, Subsurface Radar, Radio i Svjaz, Moscow (In Russian).

Morah, M. L., Greenfield, R. J. Arcone, S. A., Delaney, A. J., 2000, Multidimensional GPR array processing using Kirchgoff migration", J. Applied Geophysics, 43:281.

Nathanason, F. E., Ed., 1991, Radar Design Principles, McGraw-Hill, Inc.

Olhoeft, G. R., 2000, Maximizing the Information Return from Ground Penetrating Radar, J. of Applied Geophysics, 43:175.

Plumb, R.G., Noon D.,A., Longstaff, I. D., Stickley G. F., 1998, A Waveform-Range Performance Diagram for Ground-Penetrating Radar, J. of Applied Geophysics, 40:117. 
Ultra-Wideband, Short-Pulse Electromagnetics 5 edited by P.D. Smith and S.R. Cloude, November 2002, 199(8)

Rappaport, C. M., Reidy, D. M., 199?, Focused Array Radar for Real Time Imaging and Detection, in: Proceedings of SPIE Conference, 2747:202.

Robert A., 1998, Dielectric Permittivity of Concrete Between $50 \mathrm{MHz}$ and $1 \mathrm{GHz}$ and GPR Measurements for Building Materials Evaluation, J. of Applied Geophysics, 40: 89.

Skolnik, M. I., Ed., 1990, Radar handbook, McGraw Hill, 2nd edition.

Soumekh, M., 1999, Synthetic Aperture Radar Signal Processing with Matlab Algorithms, A Willey-Interscience Publications.

Stiles, J. M., Parra-Bocaranda, P., Apte, A., 1999, Detection of Object Symmetry Using Bistatic and Polarimetric GPR Observations, in: Proceedings of SPIE Conference on Detection and Remediation Technologies for Mines and Minelike Targets IV, 3710: 992.

Wahrus, J. P., Mast J. E., Johnson E. M., Nelson S. D., 1994, Advanced Ground Penetrating Radar, in Proceedings of SPIE Conference, 2275:177. 